ISSN 2455-2550

DOI: http://dx.doi.org/10.20431/2455-2550.0201003

www.arcjournals.org

\title{
Resveratrol: A Cardioprotective Agent
}

\author{
Shakila Banu \\ Department of Food Processing and Preservation Technology, Faculty of Engineering, \\ Avinashilingam University for Women, Coimbatore, India
}

\begin{abstract}
Today, science has a better understanding of why fruits and vegetables should be part of a healthy eating plan. Polyphenols are common constituents of the human diet, present in most foods and beverages of plant origin. They are considered to contribute to the prevention of various degenerative diseases, including cardiovascular diseases. Resveratrol is a polyphenol that occurs naturally in grapes, peanuts, and a number of other plants. It is found in foods/drinks made from grapes and peanuts, and also in a number of herbal remedies, both alone and as part of plant extracts. Resveratrol, initially used for cancer therapy, has shown beneficial effects against most degenerative and cardiovascular diseases from atherosclerosis, hypertension, and heart failure to diabetes, obesity, and aging. An attempt has been made to present the role of resveratrol in cardiovascular disease and the research challenges in this area.
\end{abstract}

Keywords: Polyphenols, resveratrol, cardiovascular diseases, grapes, research challenges etc.

\section{INTRODUCTION}

Phenolic compounds have been receiving increasing interest from consumers and manufacturers because numerous epidemiological studies have suggested associations between consumption of polyphenol-rich foods or beverages and the prevention of certain chronic diseases such as cancers and cardiovascular diseases. Cardiovascular disease (CVD), in particular coronary heart disease and stroke, is a major cause of mortality in developed nations [1].CVD is a chronic, multi-factorial disease in which a range of genetic and environmental factors plays a role in its initiation, progression and development [2]. Numerous epidemiological and human intervention studies have suggested that regular consumption of polyphenol-rich foods, such as fruits, vegetables, cocoa, tea and wine, may exert cardio-protective effects in humans [3]. Resveratrol, a dietary polyphenol may play an important role in this phenomenon. Research activities have been focused on cardioprotection by grape-derived resveratrol, in large part to address the correlation between intake of resveratrol and a reduced risk of Cardiovascular disease.

\section{RESVERATROL}

Resveratrol (3,5,4'-trihydroxy-trans stilbene) is a stilbenoid, a type of natural phenol, and a phytoalexin produced naturally by several plants when under attackby pathogens such as bacteria or fungi. Resveratrol is important in plant biology because it serves as parent molecule for a family of polymers known as viniferin. These compounds are able to inhibit the progress of fungal infections in plants [4]. Resveratrol exists as two geometric isomers: cis- $(Z)$ and trans- $(E)$ as shown in Figure 1 .

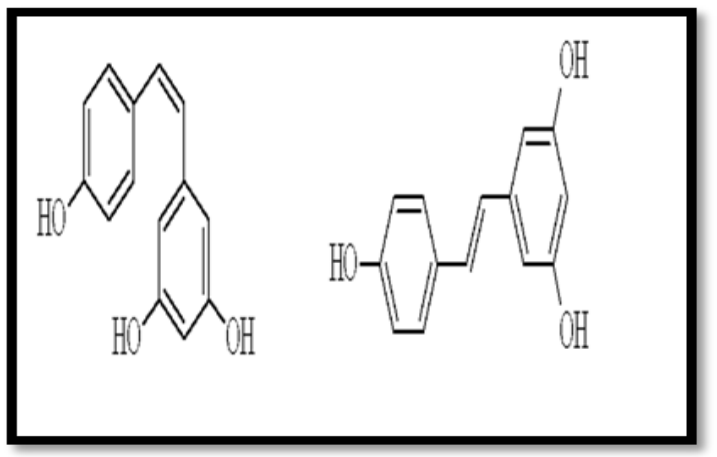

Figure 1. cis and trans isomers of resveratrol 
However trans-resveratrol is the preferred steric form and is relatively stable if protected from high $\mathrm{pH}$ and light, it is the commonly studied form of resveratrol as reported by most laboratories [5]. The trans- and cis-resveratrol can be either free or bound to glucose [6].

The trans-form can undergo isomerisation to the cis-form when exposed to ultraviolet irradiation, a process called photoisomerization [7].

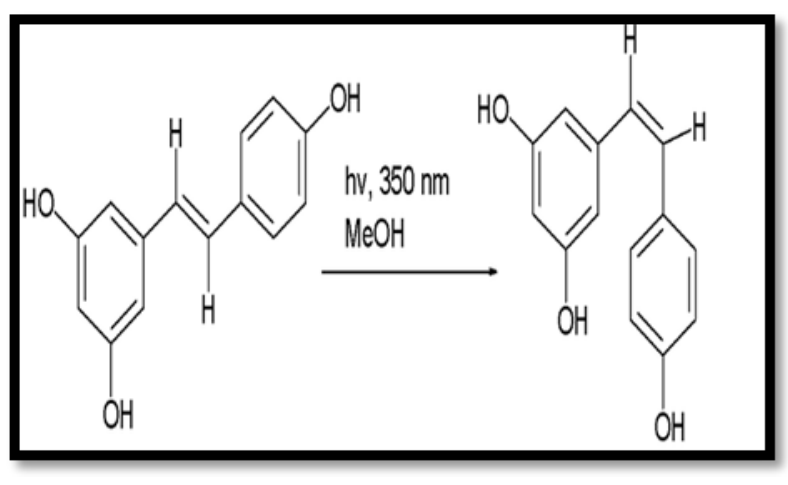

Figure 2. Resveratrol isomerization

Resveratrol is reported to have numerous biological properties and implications for human health and disease [5].

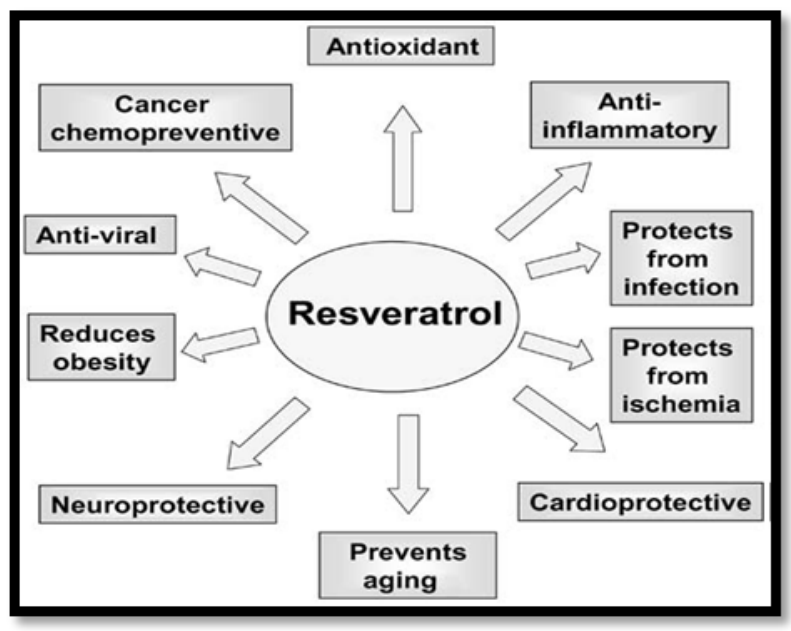

Figure 3. Health benefits of resveratrol [5]

\section{SourCes OF RESVERATrol}

Resveratrol has been detected in trees, in a few flowering plants, in peanuts, and in grapevines. The major dietary sources of resveratrol include grapes, wine, peanuts, and peanut products [8]. Fresh grape skins contain 50 to $100 \mathrm{mg}$ resveratrol per gram [5]. Red wine contains between 0.2 and $5.8 \mathrm{mg} / \mathrm{l}$ [9], depending on the grape variety, while white wine has much less, because red wine is fermented with the skins, allowing the wine to extract the resveratrol, whereas white wine is fermented after the skin has been removed [10].

The composition of wine is different from that of grapes since the extraction of resveratrols from grapes depends on the duration of the skin contact, and the resveratrol 3-glucosides are in part hydrolysed, yielding both trans-and cis-resveratrol. Muscadine grapes contain high levels of resveratrol, with multiple cardioprotective effects. These antioxidants are thought to be the effective agents behind the so-called,French Paradox, the low mortality rate from CAD amongst the French population despite their high-fat diet and smoking [11]. The concentration of resveratrol present in peanuts is dependent upon many different factors. Cultivar, growing season, weather conditions, and peanut maturity have been shown to affect resveratrol concentrations [12].

\section{Resveratrol as a CARdioprotective Agent:}

Inflammation plays a major role in the pathogenesis of a wide variety of diseases including cardiovascular diseases, cancer, diabetes, Alzheimer's disease, and autoimmune diseases. Agents that can suppress inflammation thus have a potential in mitigating the symptoms of the disease. 
Resveratrol exhibits antioxidant and antiinflammatory activities and thus may have potential in the treatment of these diseases.

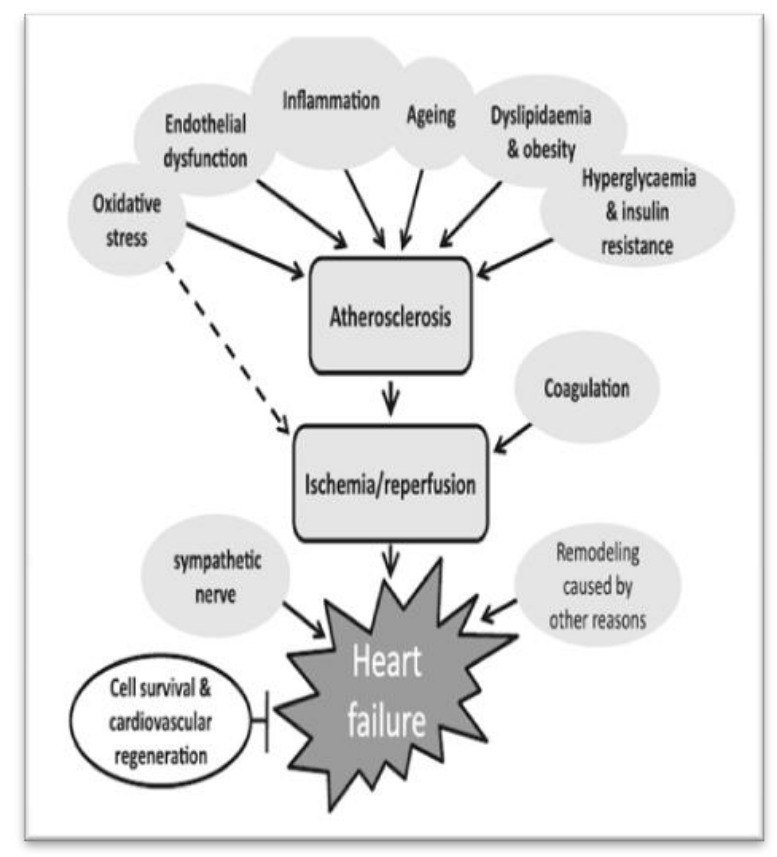

Figure 4. Multiple targets of resveratrol in cardiovascular disease [13]

Resveratrol plays an import role in maintaining stationarity of the cardiovascular system. The risk factors colored gray make coronary atherosclerosis, myocardial ischemia, and end-stage heart failure happen. Resveratrol, by interrupting these factors and events, may be possible to prevent or slow the de velopment of cardiovascular disease. In addition, resveratrol at lower dose prolongs cardiomyocytes survival in failing hearts, enhances angiogenesis under postischemic conditions, and improves cardiac stem cells homing, proliferation, and differentiation, thus may be possible to reverse heart dysfunction [13].

It achieves the effects by the following functions.

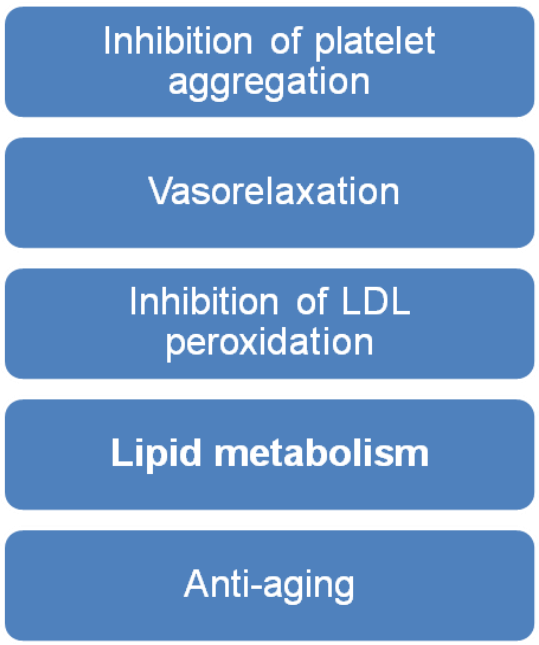

\section{Resveratrol Inhibits Platelet Activation and Aggregation}

Platelet activation has been shown to play a critical role in the pathogenesis of CAD-related complications [5]. Platelets are cells without nuclei that are made in the bone marrow and function to stop bleeding via aggregation at the wound site. Platelets can be activated by several different factors, including adenosine diphosphate (ADP), collagen, and thrombin. When activated platelets change morphology they aggregate and seal damaged blood vessels. Excessive aggregation can lead to the development of cardiovascular disease. Pretreatment of platelets with resveratrol has been shown to inhibit lipopolysaccharide (LPS) and LPS + thrombin-stimulated platelet adhesion to collagen and fibrinogen in a non-dose-dependent manner [14] 
Wang and others demonstrated that rabbits receiving resveratrol supplementation had reduced rates of platelet aggregation when fed a high cholesterol diet. Importantly, resveratrol did not change total plasma LDL levels in this study [15]. Wang's study is consistent with the findings by other investigators that resveratrol inhibited the activation and aggregation of platelets in response to thrombin, ADP, and collagen, probably through the blockade of thromboxane B2 synthesis [16]. Wang's study suggests that resveratrol administration may positively modify the outcomes of these clinical conditions.

\section{RESVERATROL IN VASORELAXATION}

The cardioprotective effects of resveratrol may also be due, in part, to its vasorelaxation properties. Chen and Pace-Asciak [17] examined the vasorelaxation effect of resveratrol on rat aortic rings with and without intact endothelium. Pretreatment with resveratrol resulted in a dose-related decrease in noradrenaline (NA)- and phenylephrine (PE)-induced contraction in endothelium-intact rat aortic rings. Endothelium-independent rings required higher concentrations of resveratrol before relaxation was observed. The authors concluded that resveratrol mediates vasorelaxation in endothelium-intact and endothelium-independent aortic rings via nitric oxide-dependent and independent mechanisms, respectively.
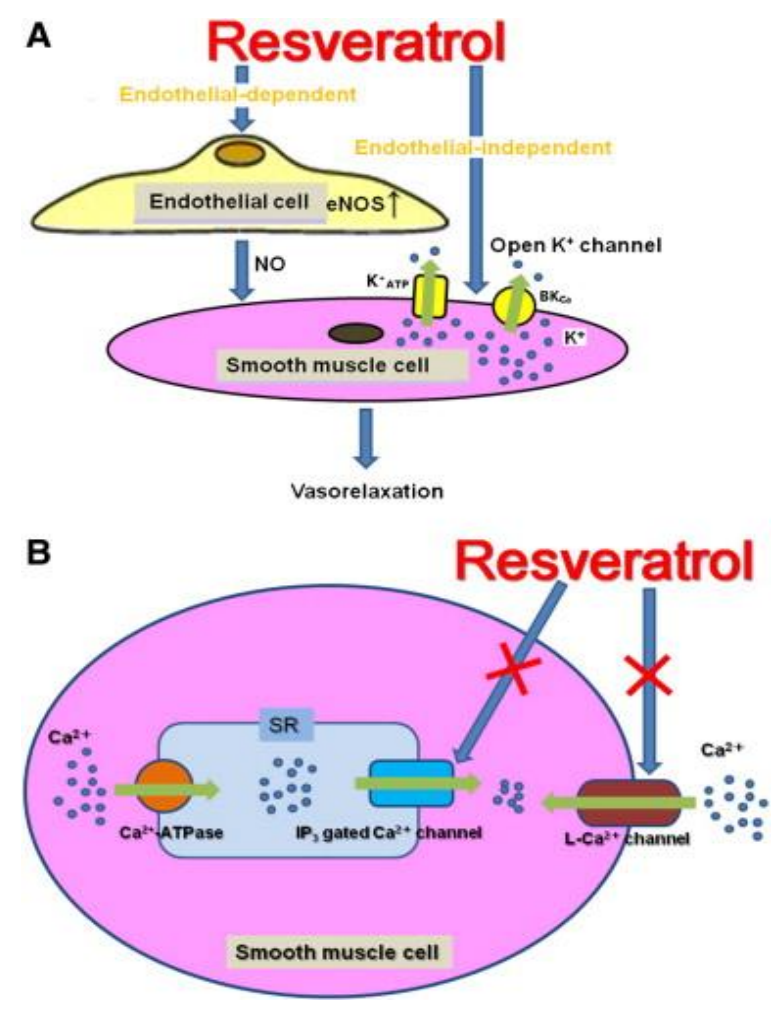

Figure 4. Vasorelaxation by resveratrol

This figure suggests that resveratrol can exert its vasorelaxing effect in both endothelial-dependent and endothelial-independent manners. Resveratrol relaxes vessel by inhibiting extracellular calcium influx and intracellular calcium release. Resveratrol contributes to the synthesis and secretion of the endothelial-dependent vasodilator NO in endothelial cells, which mediates the vasorelaxing effect of resveratrol. $\mathrm{K}_{\mathrm{ATP}}^{+}$channel and $\mathrm{BK}_{\mathrm{Ca}}$ are probably involved in vasorelaxation induced by resveratrol. Resveratrol might exert its vasorelaxing effect by opening $\mathrm{K}^{+}$channel to hyperpolarize vascular smooth muscle. It can inhibit not only the extracellular calcium influx but also intracellular calcium release in smooth muscle cells [16].

\section{RESVERATROL IN LIPID METABOLISM}

Considerable evidence suggests that oxidation of low-density lipoprotein (LDL) is a crucial step in the pathogenesis of atherosclerosis. Dietary antioxidants that can inhibit LDL oxidation are receiving considerable attention for their role in the prevention of coronary heart disease. LDL contains phospholipids, free and esterified cholesterol, triacylglycerols, and amino acids, which form apolipoprotein B. The proteins and the polyunsaturated fatty acids components of the LDL are 
susceptible to free radical-mediated oxidation, particularly if the antioxidant content of the LDL is low. When LDL is oxidized in vitro, there is a loss of polyunsaturated fatty acids yielding a range of fragments including hydroperoxides, aldehydes and ketones, which conjugate with other LDL-bound lipids and the apolipoprotein B. This minimally modified LDL has a number of properties that could increase its atherogenecity [11].

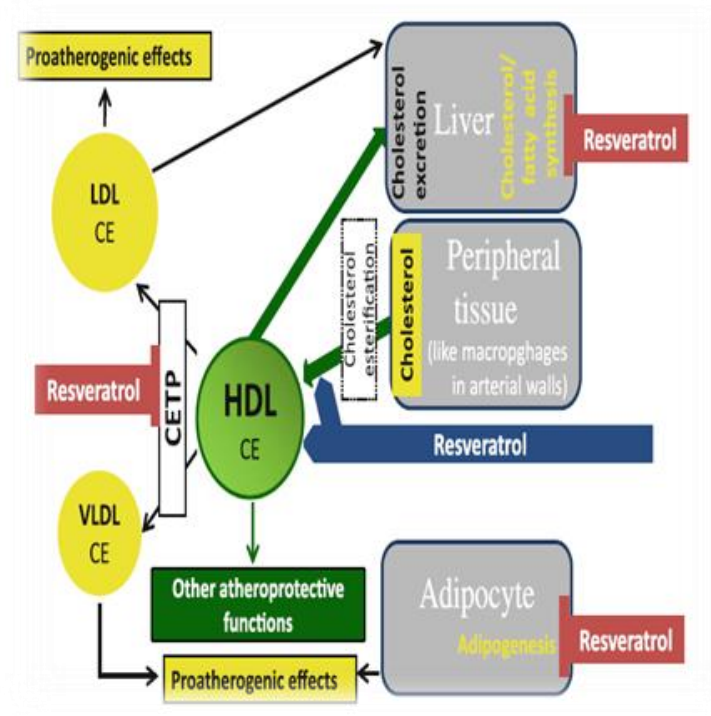

Figure 5. Resveratrol and cholesterol metabolism [13]

Resveratrol reduces cholesterol synthesis by downregulating HMG-CoA reductase. Resveratrol also enhances reverse cholesterol transport/cholesterol excretion through increasing HDL levels and the capacity of HDL to mediate cholesterol efflux from macrophages in arterial walls.

CETP is a plasma glycoprotein that facilitates the transfer of cholesteryl esters from the atheroprotective HDL to the proatherogenic LDL and VLDL [18] and resveratrol has been shown to inhibit the activity of CETP. Furthermore, adipocytes store cholesterol and have proatherogenic effects. Resveratrol can prevent adipogenesis and plays a role in dyslipidemia and obesity. LDL lowdensity

lipoprotein, CE cholesteryl ester, CETP cholesteryl ester transfer protein, HDL high-density lipoprotein, VLDL very-low-density lipoprotein [13].

Resveratrol was assumed to protect against atherosclerosis by reducing the peroxidative degradation of LDL. The in vitro effectiveness of resveratrol was thought to be mainly due to its capacity to chelate copper, although it also scavenges free radicals. Resveratrol is more effective than butylhydroxytoluene (BHT), quercetin or tocopherol on lipid peroxidation in liposomes and in rat liver. Resveratrol can inhibit LDL oxidation and it mainly acts by reducing the copper-catalyzed oxidation, while flavonoids are better scavengers of free radicals [11].

\section{Anti-Aging EfFects of Resveratrol}

Aging is a risk factor of cardiovascular diseases. To slower the speed of aging and prevent the occurrence of age associated chronic diseases is a key issue in modern society. Resveratrol can activate anti-aging genes and facilitate extending lifespan in lower organisms like yeasts and worms [19]. Resveratrol also increase survival of thehigh-calorie-diet-fed mice. However, adding resveratrol to mice fed with a normal diet did not affect the overall survival or maximum lifespan, suggesting that resveratrol might be counteracting the negative consequences of obesity and insulin resistance, thus slowing aging [19].

\section{Clinichl Trials}

Resveratrol is a food supplement for eliminating free radical and keeping healthy, but it is not into a clinical stage yet currently. Resveratrol has a short initial half-life $(* 8-14$ min for the primary molecule) and is extensively metabolized in the body. Blocking the metabolism of resveratrol, developing analogs with improved bioavailability, or searching for new and more potent compounds 
that mimic its effects will become increasingly important [20]. Fortunately, research on the structure of resveratrol is ongoing [21]. Screening the effective elements of resveratrol in vivo and determining the differences between in vivo and in vitro results are challenge for researchers.

\section{Conclusion}

Resveratrol is reported to have numerous biological properties and implications for human health and disease. However, given its low levels in food sources including red wine, it is unlikely that desired biological endpoints will be achieved from normal dietary consumption.

In addition, further understanding of the potential toxicity, health effects, bioavailability, and metabolism of resveratrol is necessary before dietary and supplement recommendations can be made.

\section{REFERENCES}

[1] WHO: Cardiovascular diseases (CVDs). Fact sheet No317. Available online: http://www.who.int/mediacentre/factsheets/fs317/en/index.html ,2009.

[2] David Vauzour, Ana Rodriguez-Mateos, Giulia Corona, Maria Jose Oruna-Concha and Jeremy P. E. Spencer, Polyphenols and Human Health: Prevention of Disease and Mechanisms of Action, Nutrients 2, 1106-1131, 2010.

[3] Arts, I.C.; Jacobs, D.R., Jr.; Harnack, L.J.; Gross, M.; Folsom, A.R. Dietary catechins in relation to coronary heart disease death among postmenopausal women, Epidemiology 12, 668-675, 2001.

[4] Sekhara Rao Basavaraju, William D. Wagner, Natural Sources of Resveratrol and Mechanisms of Action with Emphasis on Cardiovascular Disease: A Brief Review

[5] Bharat b.agarwal, Shishir shishodia Resveratrol in Health and Disease.

[6] Mattivi F, Reniero F, Korhammer S "Isolation, characterization, and evolution in red wine vinification of resveratrol monomers". J Agric Food Chem. 43 (7): 1820-3, 1995.

[7] Elyse Bernard, Philip Britz-McKibbin, Nicholas Gernigon Resveratrol Photoisomerization: An Integrative Guided-InquiryExperiment, Journal of Chemical Education Vol. 84 No. 7 ,2007.

[8] Elisabeth Wenzel, Veronika Somoza, Metabolism and bioavailability of trans-resveratrol, Molecular nutrition and food research,2005.

[9] Gu X, Creasy L, Kester A, Zeece M "Capillary electrophoretic determination of resveratrol in wines". J. Agric. Food Chem. 47 (8): 3223, 1999.

[10] Mattivi F "Solid phase extraction of trans-resveratrol from wines for HPLC analysis". Z Lebensm Unters Forsch 196 (6): 522., 1993.

[11] Sekhara Rao Basavaraju, William D. Wagner, Natural Sources of Resveratrol and Mechanisms of Action with Emphasis on Cardiovascular Disease: A Brief Review

[12] Sobolev VS, Cole RJ. trans-Resveratrol content in commercial peanuts and peanut products. J Agric Food Chem47(4):1435-9, 1999.

[13] Hong Wang ,Yue-Jin ,Yang Hai,Yan Qian Qian Zhang ,Hui Xu, Jian-Jun Li, Resveratrol in cardiovascular disease: what is known from current research, Heart Fail Rev, DOI 10.1007/s10741-011-9260-, 2011.

[14] OlasB, Wachowicz B. Resveratrol and vitamin $C$ as antioxidants in blood platelets. Thromb Res 106(2):143-8, 2002.

[15] Wang Z, Huang Y, Zou J, Cao K, Xu Y, and Wu JM, Effects of red wine and wine polyphenol resveratrol on platelet aggregation in vivo and in vitro, IntJ Mol Med 9, 77-79, 2002.

[16] Pace-Asciak CR, Hahn S, Diamandis EP, Soleas G, and Goldberg DM, The red wine phenolics trans-resveratrol and quercetin block human platelet aggregation and eicosanoid synthesis: implications for protection against coronary heart disease, Clin Chim Acta 235, 207-219, 1995.

[17] ChenC, Pace Asciak,Vaso relaxing activity of resveratrol and quercetin in isolated rat aorta. Gen Pharmacol 27(2):363-6, 1996.

[18] Weber O, Bischoff H, Schmeck C, Bo"ttcher MF,Cholesteryl ester transfer protein and its inhibition. Cell Mol Life Sci 67:3139-3149, 2010.

[19] Das DK, Mukherjee S, Ray D, Resveratrol and red wine, healthy heart and longevity. Heart Fail Rev 15:467-477, 2010 
[20] Baur JA, Sinclair DA , Therapeutic potential of resveratrol: the in vivo evidence. Nat Rev Drug Discov 5:493-506, 2006.

[21] Lagouge M, Argmann C, Gerhart-Hines Z, Meziane H, Lerin C, Daussin F, Messadeq N, Milne J, Lambert P, Elliott P, Geny B, Laakso M, Puigserver P, Auwerx J,Resveratrol improves mitochondrial function and protects against metabolic disease by activating SIRT1 and PGClalpha. Cell 127:1109-1122, 2006. 